\title{
Deficiencies and Countermeasures of Second Language Acquisition from the Perspective of Dynamic System Theory
}

\author{
Wang Sa \\ Beihua University School of Foreign Language, Jilin City, Jilin Province 132013
}

Keywords: dynamic system theory; nonlinear causality; dynamic interaction; dynamic development.

\begin{abstract}
The study of second language acquisition under the dynamic system theory is more suitable for describing the dynamics of second language acquisition because of the attention to the interaction of multiple factors in the system and the development and changes of the related factors in time. The study of second language acquisition under the dynamic system theory should first find out the group of important factors in the second language acquisition system. Firstly, test it in the positive layer. Secondly, it pays attention to the interaction between the key factors in the system and the characteristics of their development with time, and explores the regularity of the dynamic development of the system. Finally, according to the purpose of research, various research methods are creatively used to achieve scientific description or prediction of second language acquisition.
\end{abstract}

\section{Introduction}

Two language acquisition research can be divided into two main categories: one is to understand the two language learning process; the two is to explain the differences in the results of two language learning. The dynamic system theory holds that language is not fixed and a dynamic system; the development of two language can be regarded as the process of the change of the language learner's interlanguage near the target language, and there is no separation in the process of development; the learners' performance in a certain subsystem of the two language can not reflect the development of the second language; the language is not only a cognitive language, but the language is not only a cognitive language. Resources are also social resources, and the language development of learners does not continue to rise along the stage of development; the individual development path of the learners is different and has its own variation. In view of this, this study, based on the existing achievements of dynamic system theory and two language acquisition research, starts with the basic characteristics of dynamic system theory, clarifies the shortcomings of the two language acquisition research, and tries to find out the coping strategies.

\section{Basic characteristics of dynamic system theory}

The dynamic system theory has 8 important features: the sensitive dependence on the initial conditions, the complete connection, the nonlinear development, the reorganization of the system and its interaction with the environment, the dependence on the internal and external resources, the "attraction state", the repetition and the emergence of the continuity in the continuous change. The study of dynamic system theory no longer uses the hierarchical or linear model of language development, turning to the solution path that can include the internal and external determinants of the language.

Because dynamic system theory covers the complexity of the interaction of multiple factors in two language acquisition, the internal and external factors of integration of the learners, the emphasis on individual research, the emphasis on the change and development of the language system, and the elimination of the boundary of qualitative and quantitative research, it is very suitable for guiding the study of two language acquisition. In fact, in recent years it has been dynamic. Research from the perspective of system theory has been a hot topic in the field of two language acquisition. 


\section{The deficiency of the study of two language acquisition from the perspective of dynamic system theory}

First of all, because the dynamic system theory emphasizes the "complete connection" of the factors in the system rather than the specific factors, it is sometimes impossible to know how many factors affect the operation of a system or how each factor can be linked. Therefore, it is one of the most important challenges for the study of two language acquisition in the dynamic system theory to clarify which factors of infinitely many variables are "fully linked" together, and how they interact and integrate them.

Secondly, from the perspective of dynamic systems theory, L2 studies are mainly descriptive and explanatory, and there are some shortcomings in predicting language development. The main purpose of the study of two language acquisition is to understand the characteristics of language development and explore the common law of language development. In other words, the study of two language acquisition should be used to describe and explain the individual phenomenon of two language acquisition. On the other hand, the findings of the study should be used to predict the general results of language acquisition. Therefore, how to use the dynamic system theory to guide the prediction of the common law of language development is particularly important for the development of the two language research.

Thirdly, the study of second language acquisition from the perspective of dynamic system theory and from other perspectives is difficult to integrate because of the fundamental differences in language development. The dynamic system theory holds that the target language itself is not fixed, and that a single factor can not lead to a certain result of language acquisition, and a certain phenomenon in the language field can not reflect the general picture of language acquisition. Therefore, there is a great difference between the study of second language acquisition from the perspective of dynamic system theory and the traditional study, which leads to their incompatibility.

\section{Countermeasures}

\subsection{Determine the combination of important factors in the two language acquisition system.}

The complexity of the dynamic system leads to the difficulty of carrying out the empirical study. First, it is necessary to separate the highly related factors from many factors of the two language acquisition system to reduce the complexity of the system, and then investigate the dynamics of these factors, that is, to find out the key to the effect of the two language acquisition from the two language acquisition system. Factors and how they interact bring about new features in the language system.

There are two feasible ways to sort out the key factors restricting the development of the system. First, the cluster analysis method is used to find out which factors are usually combined together on the basis of the literature research, and how they work together to lead to the specific results of language development. Secondly, the qualitative modeling method of backtracking is used to find out why some components of the system lead to a certain result, for example, from the typical class of the learners to trace the reasons for the formation of typical categories.

\subsection{The interaction between important factors in the research system}

In response to the complexity of multi factor interaction in a dynamic system, researchers should not only explore which key factors are often combined, but also focus on how they interact. There is no doubt that two language acquisition is more likely to be influenced by the multifactor interaction complexity than the mother tongue acquisition, so it is more effective to explain the dynamic perspective of two language acquisition than to adopt the linear attribution perspective. The interpretation of two language acquisition effectiveness and the construction of the two language acquisition model should not only consider the multi factor role of the complex system of two language acquisition, but also consider the dynamic interaction between the subsystems. The interaction of environment, learners and language factors runs through the whole process of interlanguage development, which affects the input, absorption, reconstruction, integration and 
output of the two language acquisition, and ultimately determines the results of the two language acquisition.

\subsection{The dynamic change track and regularity of language factors and learner factors in the research system.}

Dynamic system theory is more successful in describing and interpreting the unpredictability of the system, individual differences, and two language fluency (Segalowitz 2010), which mainly covers the dynamic changes in the two subsystems of the two language and the learners' differences. Therefore, it is no problem to apply this theory to the study of second language acquisition. It is important for researchers to choose the appropriate theoretical paradigm according to the purpose of the study.

\subsubsection{The dynamic changes of the two language in time}

The study of two language acquisition based on dynamic system theory should not only consider the interactivity between different factors in a specific environment, but also consider the changes in the development of various factors in the system. In order to study the variability of the factors in the system, it is necessary to consider the initial state of the system, the continuity of the change and the "state of attraction", the repeatability of the change, the emergence of the change and so on. The learners' initial level will have a critical impact on subsequent learning. The level of specific language areas may be developed over time, while the language level in other areas may be eroded, and the development of language has a "state of attraction".

\subsubsection{The dynamic changes of individual differences in time}

Individual differences in learning motivation, cognition and emotion will vary with time, such as the initial two language motivation and attitude that affect subsequent learning interest, strategies, methods, and so on. So far, there are still few empirical studies on the dynamic change of learner's individual differences from a dynamic system perspective. By describing the dynamic changes of language factors and learners' factors in the two language acquisition system, the researchers can find the characteristics and development trajectory of the two language changes, and then find the law of language development. Therefore, these descriptive studies are not only valuable, but also the basis of predictive research.

\subsection{Creative use of a variety of new methods}

The research methods of two language acquisition in the dynamic system theory can be summed up in four categories: the first kind uses the diachronic dynamic description method to collect the intensive data for analysis; the second classes form a more scientific hypothesis on the basis of dynamic description, then the qualitative modeling of the related data, and the third category using the retrospective qualitative modeling method, The differences between qualitative modeling of diachronic materials and qualitative modeling of backtracking materials are compared, and the fourth kinds of comprehensive use of observation, interview, questionnaire survey and measurement are used to carry out case study. In conclusion, researchers in this field have explored a variety of research methods. However, because each method has different aims and advantages and disadvantages, the researchers need to carefully identify, adjust or optimize the existing and potential research methods, and then creatively apply them to meet the needs of the research. And scientifically describe or predict two language acquisition.

\section{Conclusion}

Compared with the traditional two language acquisition research, the study of two language acquisition under the dynamic system theory uses a multi factor dynamic interaction view, focusing on the dynamic development of different levels of two language acquisition at different levels, and thus is more suitable for the description of the two language acquisition. The study of two language acquisition based on dynamic system theory can also provide a very useful reference for foreign 
language teaching. From the view of the development of the two language, the factors are also dynamic in time, including the development and change of each factor itself and the dynamic changes between the various factors. Therefore, it is necessary to make full use of the changing laws of different factors in a given time, and lead to the emergence of new characteristics. If we can make full use of the dynamic interaction of multiple factors in the two language system and the law of the development and change of the system in the teaching, it will be more helpful to improve the efficiency and effect of teaching.

\section{Acknowledgements}

Foundation: The Research of Second Language Acquisition on the Basis of Dynamic System

Funding: Beihua University Young Teachers Upgrading Scheme Special Funding and Jilin Province Department of Education 13th Five - Year Plan, Fund Number: (JJKH20180402)

\section{References}

[1] Dai Yuncai. Motivation of Second Language Learning from the Perspective of Complex Dynamic System Theory [J]. Foreign Language Studies, 2015, (6): 72-80.

[2] Dai Yuncai, Yang Lianrui. The Integrated Model of Second Language Acquisition and Its Dynamics [J]. Foreign Language Teaching, 2013, (6): 49-53.

[3] Li Lanxia. Dynamic system theory and second language development [J]. Foreign language teaching and research, 2011, (3): 409 - 421.

[4] Xu Xiyang, Wu Yongyi. Complex Dynamic Systems Theory: Reflections on Second Language Acquisition Research [J]. Language Teaching and Research, 2015, (2): 1-7.

[5] Zheng Yongrong. A Study of the Deep Development of Second Language Vocabulary within the Framework of Dynamic Systems Theory [J]. Foreign Language Education in China, 2014, (3): 62-73. 